\title{
AN ALGORITHM TO VERIFY LOCAL THRESHOLD TESTABILITY OF DETERMINISTIC FINITE AUTOMATA
}

\author{
A.N. Trahtman \\ Bar-Ilan University, Dep. of Math. and CS, 52900,Ramat Gan, Israel \\ Lecture Notes in Computer Scence 2214(2001), 164-173 \\ email:trakht@macs.biu.ac.il
}

\begin{abstract}
A locally threshold testable language $L$ is a language with the property that for some nonnegative integers $k$ and $l$, whether or not a word $u$ is in the language $L$ depends on (1) the prefix and suffix of the word $u$ of length $k-1$ and (2) the set of intermediate substrings of length $k$ of the word $u$ where the sets of substrings occurring at least $j$ times are the same, for $j \leq l$. For given $k$ and $l$ the language is called $l$-threshold $k$-testable. A finite deterministic automaton is called $l$-threshold $k$-testable if the automaton accepts a $l$-threshold $k$-testable language.

In this paper, the necessary and sufficient conditions for an automaton to be locally threshold testable are found. We introduce the first polynomial time algorithm to verify local threshold testability of the automaton based on this characterization.

New version of polynomial time algorithm to verify the local testability will be presented too.
\end{abstract}

Key words:deterministic finite automaton, locally threshold testable, algorithm, semigroup

AMS subject classification 68Q25, 68Q45, 68Q68, 20M07

\section{Introduction}

The concept of local testability was introduced by McNaughton and Papert [20] and by Brzozowski and Simon [7]. Local testability can be considered as a special case of local $l$-threshold testability for $l=1$. Locally testable languages, automata and semigroups have been investigated from different points of view (see [6] - [17], [19], [22], [28] - [36]). In [21], local testability was discussed in terms of "diameterlimited perceptrons". Locally testable languages are a generalization of the definite and reverse-definite languages, which can be found, for example, in [11] and [23]. Some variations of the concept of local testability (strictly, strongly) obtained by changing or omitting prefixes and suffixes in the definition of the concept were studied in [6], [8], [20], [22], [28].

Locally testable automata have a wide spectrum of applications. Regular languages and picture languages can be described by a strictly locally testable languages [6], [14]. Local automata (a kind of locally testable automata) are heavily used to construct transducers and coding schemes adapted to constrained channels [3]. Literal morphisms may be modelled by help of 2-testable languages [9]. In [13], locally testable languages are used in the study of DNA and informational macromolecules in biology.

Kim, McNaughton and McCloskey ([15], [16]) have found necessary and sufficient conditions of local testability and a polynomial time algorithm for local testability problem based on these conditions. The realization of the algorithm is described by Caron in [8]. A polynomial time algorithm for local testability problem for semigroups was presented in [29].

The locally threshold testable languages were introduced by Beauquier and Pin [4]. These languages generalize the concept of locally testable language and have been studied extensively in recent years (see [5], [22], [27], [33], [34]), [25].

An important reason to study locally threshold testable languages is the possibility of being used in pattern recognition [10], [12], [25]. Stochastic locally threshold testable languages, also known as $N$-grams 
are used in pattern recognition, particulary in speech recognation, both in acoustic-phonetics decoding as in language modeling [32]. An application of these languages to the inference problem considered in [25]. The syntactic characterization of locally threshold testable languages one can find in [4]:

Given the syntactic semigroup $S$ of the language $L$, we form a graph G(S) as follows. The vertices of $\mathrm{G}(\mathrm{S})$ are the idempotents of $S$, and the edges from $e$ to $f$ are the elements of the form esf. A language $L$ is locally threshold testable if and only if $S$ is aperiodic and for any two nodes $e, f$ and three edges $p$, $q, r$ such that $p$ and $q$ are edges from $e$ to $f$ and $r$ is an edge from $f$ to $e$ we have

$$
\text { prq }=\text { qrp }
$$

Since only five elements of the semigroup $S$ are considered, there exists a polynomial time algorithm of order $O\left(|S|^{5}\right)$ for local threshold testability problem in the case of semigroups. But the cardinality of the syntactic semigroup of a locally threshold testable automaton is not polynomial in the number of its nodes [16]. This is why the study of the automaton and the state transition graph of the automaton is important from the practical point of view (see [16], [17]) and we use here this approach.

For the state transition graph $\Gamma$ of an automaton, we consider some subgraphs of the cartesian product $\Gamma \times \Gamma$ and $\Gamma \times \Gamma \times \Gamma$. In this way, necessary and sufficient conditions for a deterministic finite automaton to be locally threshold testable are found. We present here $O\left(n^{5}\right)$ time algorithm to verify local threshold testability of the automaton based on this characterization.

Necessary and sufficient conditions of local testability from [16] are considered in this paper in terms of reachability in the graph $\Gamma \times \Gamma$. New version of $O\left(n^{2}\right)$ time algorithm to verify local testability based on this approach will be presented too.

\section{Notation and definitions}

Let $\Sigma$ be an alphabet and let $\Sigma^{+}$denote the free semigroup on $\Sigma$. If $w \in \Sigma^{+}$, let $|w|$ denote the length of $w$. Let $k$ be a positive integer. Let $i_{k}(w)\left[t_{k}(w)\right.$ ] denote the prefix [suffix] of $w$ of length $k$ or $w$ if $|w|<k$. Let $F_{k, j}(w)$ denote the set of factors of $w$ of length $k$ with at least $j$ occurrences. A language $L$ [a semigroup $S$ ] is called l-threshold k-testable if there is an alphabet $\Sigma$ [and a surjective morphism $\left.\phi: \Sigma^{+} \rightarrow S\right]$ such that for all $u, v \in \Sigma^{+}$, if $i_{k-1}(u)=i_{k-1}(v), t_{k-1}(u)=t_{k-1}(v)$ and $F_{k, j}(u)=F_{k, j}(v)$ for all $j \leq l$, then either both $u$ and $v$ are in $L$ or neither is in $L[u \phi=v \phi]$.

An automaton is $l$-threshold $k$-testable if the automaton accepts a $l$-threshold $k$-testable language [the syntactic semigroup of the automaton is $l$-threshold $k$-testable].

A language $L$ [a semigroup $S$, an automaton $\mathbf{A}$ ] is locally threshold testable if it is $l$-threshold $k$-testable for some $k$ and $l$.

A semigroup without non-trivial subgroups is called aperiodic [4]

$|\Gamma|$ denotes the number of nodes of the graph $\Gamma$.

$\Gamma^{i}$ denotes the direct product of $i$ copies of the graph $\Gamma$.

A maximal strongly connected component of the graph will be denoted for brevity as SCC [15], a finite deterministic automaton will be denoted as DFA [16]. A node from an $S C C$ will be called for brevity as an SCC - node.

If an edge $\mathbf{p} \rightarrow \mathbf{q}$ is labeled by $\sigma$ then let us denote the node $\mathbf{q}$ as $\mathbf{p} \sigma$.

We shall write $\mathbf{p} \succeq \mathbf{q}$ if the node $\mathbf{q}$ is reachable from the node $\mathbf{p}$ or $\mathbf{p}=\mathbf{q}$.

In the case $\mathbf{p} \succeq \mathbf{q}$ and $\mathbf{q} \succeq \mathbf{p}$ we write $\mathbf{p} \sim \mathbf{q}$ (that is $\mathbf{p}$ and $\mathbf{q}$ belong to one $S C C$ or $\mathbf{p}=\mathbf{q}$ ).

\section{The necessary and sufficient conditions}

Let us formulate the result of Beauquier and Pin [4] in the following form: 
Theorem 11 [4] A language $L$ is locally threshold testable if and only if the syntactic semigroup $S$ of $L$ is aperiodic and for any two idempotents $e, f$ and elements $a, u, b$ of $S$ we have

$$
\text { eafuebf }=\text { ebfueaf }
$$

Let us recall the concept of implicit operation [24], [2]: The unary operation $x^{\omega}$ assigns to every element $x$ of a finite semigroup the unique idempotent in the subsemigroup generated by $x$.

The set of locally threshold testable semigroups forms a pseudovariety of semigroups ([33], [4]). So the theorem 11 implies

Corollary 12 The pseudovariety of locally threshold testable semigroups consists of aperiodic semigroups and satisfies the pseudoidentity

$$
x^{\omega} y z^{\omega} u x^{\omega} t z^{\omega}=x^{\omega} t z^{\omega} u x^{\omega} y z^{\omega}
$$

Lemma 13 Let the node (p, $\mathbf{q})$ be an SCC-node of $\Gamma^{2}$ of a locally threshold testable DF A with state transition graph $\Gamma$ and suppose that $\mathbf{p} \sim \mathbf{q}$.

Then $\mathbf{p}=\mathbf{q}$.

Proof. The transition semigroup $S$ of the automaton is finite and aperiodic [18]. Suppose that for some element $e \in S$ and for some states $\mathbf{q}$ and $\mathbf{p}$ from $S C C X$ we have $\mathbf{q} e=\mathbf{q}$ and $\mathbf{p} e=\mathbf{p}$. In view of $\mathbf{q} e^{i}=\mathbf{q}, \mathbf{p} e^{i}=\mathbf{p}$ and finiteness of $S$ we can assume $e$ is an idempotent. In the $S C C X$ for some $a$, $b$ from $S$ we have $\mathbf{p} a=\mathbf{q}$ and $\mathbf{q} b=\mathbf{p}$. Hence, $\mathbf{p} e a e=\mathbf{q}, \mathbf{q} e b e=\mathbf{p}$. So $\mathbf{p} e a e b e=\mathbf{p}=\mathbf{p}(\text { eaebe })^{i}$ for any integer $i$. There exists a natural number $n$ such that in the aperiodic semigroup $S$ we have $(e a e)^{n}=(e a e)^{n+1}$. From theorem 11 it follows that for the idempotent e, eaeebe = ebeeae. We have $\mathbf{p}=\mathbf{p e a e b e}=\mathbf{p}(\text { eaeebe })^{n}=\mathbf{p}(\text { eae })^{n}(e b e)^{n}=\mathbf{p}(e a e)^{n+1}(e b e)^{n}=\mathbf{p}(e a e)^{n}(e b e)^{n}$ eae $=\mathbf{p} e a e=\mathbf{q}$. So $\mathbf{p}=\mathbf{q}$

Theorem 14 For DFA A with state transition graph $\Gamma$ the following three conditions are equivalent:

1)A is locally threshold testable.

2)If the nodes $\left(\mathbf{p}, \mathbf{q}_{1}, \mathbf{r}_{1}\right)$ and $\left(\mathbf{q}, \mathbf{r}, \mathbf{t}_{1}, \mathbf{t}\right)$ are $S C C$-nodes of $\Gamma^{3}$ and $\Gamma^{4}$, correspondingly, and

$(\mathbf{q}, \mathbf{r}) \succeq\left(\mathbf{q}_{1}, \mathbf{r}_{1}\right),\left(\mathbf{p}, \mathbf{q}_{1}\right) \succeq(\mathbf{r}, \mathbf{t}),\left(\mathbf{p}, \mathbf{r}_{1}\right) \succeq\left(\mathbf{q}, \mathbf{t}_{1}\right)$ holds in $\Gamma^{2}$

then $\mathbf{t}=\mathbf{t}_{1}$.

3) If the node $(\mathbf{u}, \mathbf{v})$ is an $S C C$-node of the graph $\Gamma^{2}$ and $\mathbf{u} \sim \mathbf{v}$ then $\mathbf{u}=\mathbf{v}$.

If the nodes $\left(\mathbf{p}, \mathbf{q}_{1}, \mathbf{r}_{1}\right),(\mathbf{q}, \mathbf{r}, \mathbf{t}),\left(\mathbf{q}, \mathbf{r}, \mathbf{t}_{1}\right)$ are $S C C$-nodes of the graph $\Gamma^{3}$ and

$(\mathbf{q}, \mathbf{r}) \succeq\left(\mathbf{q}_{1}, \mathbf{r}_{1}\right),\left(\mathbf{p}, \mathbf{q}_{1}\right) \succeq(\mathbf{r}, \mathbf{t}),\left(\mathbf{p}, \mathbf{r}_{1}\right) \succeq\left(\mathbf{q}, \mathbf{t}_{1}\right)$ hold in $\Gamma^{2}$, then $\mathbf{t} \sim \mathbf{t}_{1}$.

2)

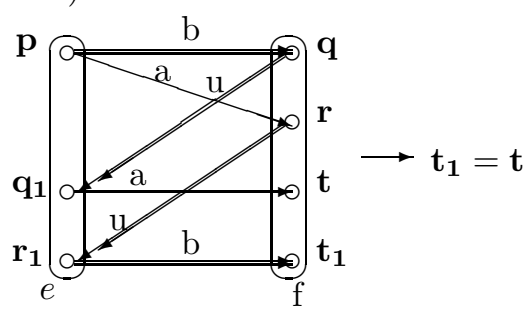

Proof. 2) $\rightarrow$ 1):

Let us consider the nodes zebfueaf and zeafuebf where $\mathbf{z}$ is an arbitrary node of $\Gamma, a, u, b$ are arbitrary elements from transition semigroup $S$ of the automaton and $e, f$ are arbitrary idempotents from $S$. Let us denote

$$
\mathbf{z} e=\mathbf{p}, \mathbf{z} e b f=\mathbf{q}, \mathbf{z} e a f=\mathbf{r}, \mathbf{z e a f u e}=\mathbf{r}_{1}, \mathbf{z} e b f u e=\mathbf{q}_{1}, \mathbf{z} e b f u e a f=\mathbf{t}, \mathbf{z e a f u e b f}=\mathbf{t}_{1} .
$$


By condition 2), we have $\mathbf{t}=\mathbf{t}_{1}$, whence zebfueaf $=\mathbf{z e a f u e b f}$. Thus, the condition eafuebf= ebfueaf (1) holds for the transition semigroup $S$. By theorem 11, the automaton is locally threshold testable.

1) $\rightarrow 3)$ :

If the node $(\mathbf{u}, \mathbf{v})$ belongs to some $S C C$ of the graph $\Gamma^{2}$ and $\mathbf{u} \sim \mathbf{v}$ then by lemma 13 local threshold testability implies $\mathbf{u}=\mathbf{v}$.

The condition eafuebf $=$ ebfueaf ((1), theorem 11) holds for the transition semigroup $S$ of the automaton. Let us consider nodes $\mathbf{p}, \mathbf{q}, \mathbf{r}, \mathbf{t}, \mathbf{q}_{1}, \mathbf{r}_{1}, \mathbf{t}_{1}$ satisfying the condition 3). Suppose

$$
\left(\mathbf{p}, \mathbf{q}_{1}, \mathbf{r}_{1}\right) e=\left(\mathbf{p}, \mathbf{q}_{1}, \mathbf{r}_{1}\right),(\mathbf{q}, \mathbf{r}, \mathbf{t}) f_{2}=(\mathbf{q}, \mathbf{r}, \mathbf{t}),\left(\mathbf{q}, \mathbf{r}, \mathbf{t}_{1}\right) f_{1}=\left(\mathbf{q}, \mathbf{r}, \mathbf{t}_{1}\right)
$$

for some idempotents $e, f_{1}, f_{2} \in S$, and

$$
\left(\mathbf{p}, \mathbf{q}_{1}\right) a=(\mathbf{r}, \mathbf{t}),\left(\mathbf{p}, \mathbf{r}_{1}\right) b=\left(\mathbf{q}, \mathbf{t}_{1}\right)(\mathbf{q}, \mathbf{r}) u=\left(\mathbf{q}_{1}, \mathbf{r}_{1}\right)
$$

for some elements $a, b, u \in S$. Then pea $f_{2}=\mathbf{p} e a f_{1}$ and $\mathbf{p} e b f_{2}=\mathbf{p} e b f_{1}$.

We have $\mathbf{t}_{1} f_{2}=$ pea $f_{1} u e b f_{1} f_{2}$. By theorem 11 , peb $f_{j} u e a f_{j}=$ pea $f_{j} u e b f_{j}$ for $j=1,2$. So we have $\mathbf{t}_{1} f_{2}=$ pea $f_{1} u e b f_{1} f_{2}=$ peb $f_{1} u e a f_{1} f_{2}$. In view of pebf $f_{2}=\mathbf{p} e b f_{1}$ and $f_{i}=f_{i} f_{i}$ we have $\mathbf{t}_{1} f_{2}=$ $\mathbf{p e b} f_{2} f_{2}$ uea $f_{1} f_{2}$. By theorem $11, \mathbf{t}_{1} f_{2}=\mathbf{p} e\left(b f_{2}\right) f_{2} u e\left(a f_{1}\right) f_{2}=\mathbf{p} e\left(a f_{1}\right) f_{2} u e\left(b f_{2}\right) f_{2}$. Now in view of pea $f_{2}=$ pea $f_{1}$ let us exclude $f_{1}$ and obtain $\mathbf{t}_{1} f_{2}=$ pea $f_{2} u e b f_{2}=\mathbf{t}$. So $\mathbf{t}_{1} f_{2}=\mathbf{t}$. Analogously, $\mathbf{t} f_{1}=\mathbf{t}_{1}$.

Hence, $\mathbf{t}_{1} \sim \mathbf{t}$. Thus 3 ) is a consequence of 1 ).

$3) \rightarrow 2)$ :

Suppose that $\left(\mathbf{p}, \mathbf{q}_{1}, \mathbf{r}_{1}\right) e=\left(\mathbf{p}, \mathbf{q}_{1}, \mathbf{r}_{1}\right),\left(\mathbf{q}, \mathbf{r}, \mathbf{t}, \mathbf{t}_{1}\right) f=\left(\mathbf{q}, \mathbf{r}, \mathbf{t}, \mathbf{t}_{1}\right)$, for some idempotents $e, f$ from transition semigroup $S$ of the automaton and

$$
\left(\mathbf{p}, \mathbf{q}_{1}\right) a=(\mathbf{r}, \mathbf{t}),\left(\mathbf{p}, \mathbf{r}_{1}\right) b=\left(\mathbf{q}, \mathbf{t}_{1}\right),(\mathbf{q}, \mathbf{r}) u=\left(\mathbf{q}_{1}, \mathbf{r}_{1}\right)
$$

for some elements $a, u, b \in S$. Therefore

$$
\begin{aligned}
& \left(\mathbf{p}, \mathbf{q}_{1}\right) \text { eaf }=\left(\mathbf{p}, \mathbf{q}_{1}\right) a f=(\mathbf{r}, \mathbf{t}) \\
& \left(\mathbf{p}, \mathbf{r}_{1}\right) \text { ebf }=\left(\mathbf{p}, \mathbf{r}_{1}\right) b f=\left(\mathbf{q}, \mathbf{t}_{1}\right) \\
& (\mathbf{q}, \mathbf{r}) u=(\mathbf{q}, \mathbf{r}) \text { fue }=\left(\mathbf{q}_{1}, \mathbf{r}_{1}\right)
\end{aligned}
$$

for idempotents $e, f$ and elements $a, u, b \in S$.

For $f=f_{1}=f_{2}$ from 3$)$ we have $\mathbf{t} \sim \mathbf{t}_{1}$. Notice that $\left(\mathbf{t}_{1}, \mathbf{t}\right) f=\left(\mathbf{t}_{1}, \mathbf{t}\right)$. The node $\left(\mathbf{t}_{1}, \mathbf{t}\right)$ belongs to some $S C C$ of the graph $\Gamma^{2}$ and $\mathbf{t} \sim \mathbf{t}_{1}$, whence by by lemma $13, \mathbf{t}=\mathbf{t}_{1}$.

Lemma 15 Let the nodes $\left(\mathbf{q}, \mathbf{r}, \mathbf{t}_{1}\right)$ and $\left(\mathbf{q}, \mathbf{r}, \mathbf{t}_{2}\right)$ be $S C C$-nodes of the graph $\Gamma^{3}$ of a locally threshold testable DF A with state transition graph $\Gamma$. Suppose that $\left(\mathbf{p}, \mathbf{r}_{\mathbf{1}}\right) \succeq\left(\mathbf{q}, \mathbf{t}_{1}\right),\left(\mathbf{p}, \mathbf{r}_{\mathbf{1}}\right) \succeq\left(\mathbf{q}, \mathbf{t}_{2}\right)$ in the graph $\Gamma^{2}$ and $\mathbf{p} \succeq \mathbf{r} \succeq \mathbf{r}_{1}$.

Then $\mathbf{t}_{1} \sim \mathbf{t}_{2}$.

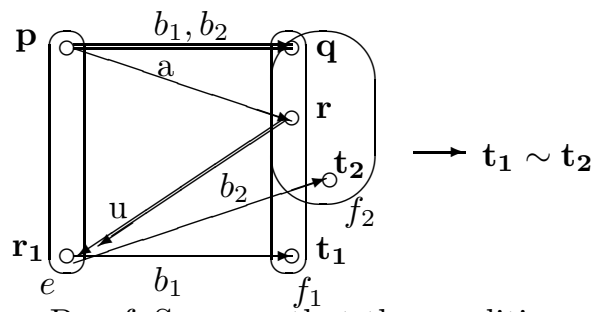

Proof. Suppose that the conditions of the lemma hold but $\mathbf{t}_{1} \nsim \mathbf{t}_{2}$.

We have $\left(\mathbf{p}, \mathbf{r}_{1}\right) e=\left(\mathbf{p}, \mathbf{r}_{1}\right),\left(\mathbf{q}, \mathbf{r}, \mathbf{t}_{1}\right) f_{1}=\left(\mathbf{q}, \mathbf{r}, \mathbf{t}_{1}\right),\left(\mathbf{q}, \mathbf{r}, \mathbf{t}_{2}\right) f_{2}=\left(\mathbf{q}, \mathbf{r}, \mathbf{t}_{2}\right)$, for some idempotents $e, f_{2}$, $f_{2}$ from the transition semigroup $S$ of the automaton and

for some elements $a, u, b_{1}, b_{2} \in S$.

$$
\left(\mathbf{p}, \mathbf{r}_{1}\right) b_{1}=\left(\mathbf{q}, \mathbf{t}_{1}\right),\left(\mathbf{p}, \mathbf{r}_{1}\right) b_{2}=\left(\mathbf{q}, \mathbf{t}_{2}\right), \mathbf{p} a=\mathbf{r}, \mathbf{r} u=\mathbf{r}_{1}
$$

If $\mathbf{t}_{1} f_{2} \sim \mathbf{t}_{2}$ and $\mathbf{t}_{\mathbf{2}} f_{1} \sim \mathbf{t}_{1}$ then $\mathbf{t}_{\mathbf{2}} \sim \mathbf{t}_{1}$ in spite of our assumption. Therefore let us assume for instance that $\mathbf{t}_{\mathbf{1}} \not \mathbf{t}_{2} f_{1}$. (And so $\mathbf{t}_{\mathbf{1}} \neq \mathbf{t}_{2} f_{1}$ ). This gives us an opportunity to consider $\mathbf{t}_{2} f_{1}$ instead of $\mathbf{t}_{2}$. So let us denote $\mathbf{t}_{2}=\mathbf{t}_{2} f_{1}, f=f_{1}=f_{2}$. Then $\mathbf{t}_{2} f=\mathbf{t}_{2}, \mathbf{t}_{1} f=\mathbf{t}_{1}$ and $\mathbf{t}_{\mathbf{1}} \nsim \mathbf{t}_{2}$. Now

$$
\mathbf{p} e a f=\mathbf{p} a f=\mathbf{r}
$$


So we have

$$
\begin{gathered}
\left(\mathbf{p}, \mathbf{r}_{1}\right) e b_{1} f=\left(\mathbf{p}, \mathbf{r}_{1}\right) b_{1} f=\left(\mathbf{q}, \mathbf{t}_{1}\right) \\
\left(\mathbf{p}, \mathbf{r}_{1}\right) e b_{2} f=\left(\mathbf{p}, \mathbf{r}_{1}\right) b_{2} f=\left(\mathbf{q}, \mathbf{t}_{2}\right) \\
\mathbf{r} u=\mathbf{r} u e=\mathbf{r}_{1}
\end{gathered}
$$

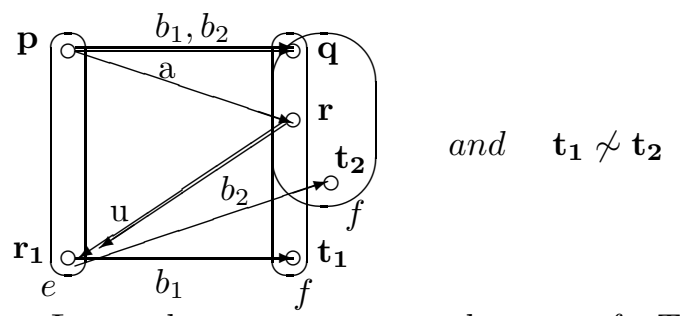

Let us denote $\mathbf{q}_{1}=\mathbf{q} u e$ and $\mathbf{t}=\mathbf{q}_{1} a f_{1}$. Then

$$
\left(\mathbf{p}, \mathbf{q}_{1}, \mathbf{r}_{1}\right) e=\left(\mathbf{p}, \mathbf{q}_{1}, \mathbf{r}_{1}\right),(\mathbf{q}, \mathbf{r}) u e=\left(\mathbf{q}_{1}, \mathbf{r}_{1}\right),\left(\mathbf{q}, \mathbf{r}, \mathbf{t}, \mathbf{t}_{i}\right) f=\left(\mathbf{q}, \mathbf{r}, \mathbf{t}, \mathbf{t}_{i}\right)
$$

So the node $\left(\mathbf{p}, \mathbf{q}_{1}, \mathbf{r}_{1}\right)$ is an $S C C$-node of the graph $\Gamma^{3}$, the nodes $\left(\mathbf{p}, \mathbf{q}, \mathbf{r}, \mathbf{t}_{i}\right)$ are $S C C$-nodes of the graph $\Gamma^{4}$ for $i=1,2$ and we have $(\mathbf{q}, \mathbf{r}) \succeq\left(\mathbf{q}_{1}, \mathbf{r}_{1}\right),\left(\mathbf{p}, \mathbf{q}_{1}\right) \succeq(\mathbf{r}, \mathbf{t})$ and $\left(\mathbf{p}, \mathbf{r}_{1}\right) \succeq\left(\mathbf{q}, \mathbf{t}_{i}\right)$ for $i=1,2$.

Therefore, by theorem 14, (2), we have $\mathbf{t}_{1}=\mathbf{t}$ and $\mathbf{t}_{2}=\mathbf{t}$. Hence, $\mathbf{t}_{1} \sim \mathbf{t}_{2}$, contradiction.

Définition 16 For any four nodes $\mathbf{p}, \mathbf{q}, \mathbf{r}, \mathbf{r}_{1}$ of the graph $\Gamma$ of a DFA such that $\mathbf{p} \succeq \mathbf{r} \succeq \mathbf{r}_{1}, \mathbf{p} \succeq \mathbf{q}$ and the nodes $\left(\mathbf{p}, \mathbf{r}_{1}\right),(\mathbf{q}, \mathbf{r})$ are $S C C$-nodes, let $T_{S C C}\left(\mathbf{p}, \mathbf{q}, \mathbf{r}, \mathbf{r}_{1}\right)$ be the $S C C$ of $\Gamma$ containing

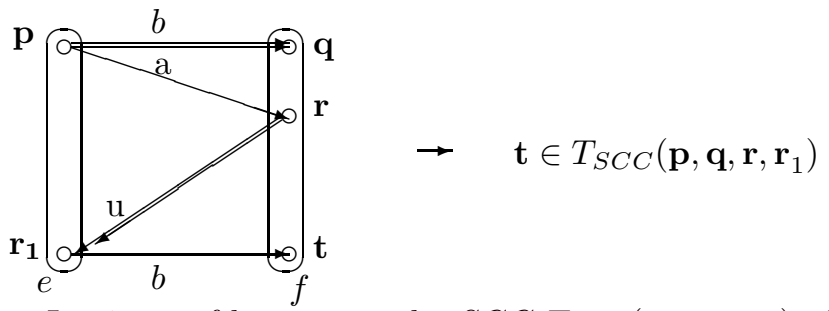

In virtue of lemma 15 , the $S C C T_{S C C}\left(\mathbf{p}, \mathbf{q}, \mathbf{r}, \mathbf{r}_{1}\right)$ of a locally threshold testable $D F A$ is well defined (but empty if the set $T\left(\mathbf{p}, \mathbf{q}, \mathbf{r}, \mathbf{r}_{1}\right)$ is empty). Lemma 15 and theorem 14 (3) imply the following theorem

Theorem 17 A DFA A with state transition graph $\Gamma$ is locally threshold testable iff

1)for every $S C C$-node $(\mathbf{p}, \mathbf{q})$ of $\Gamma^{2} \mathbf{p} \sim \mathbf{q}$ implies $\mathbf{p}=\mathbf{q}$

and

2)for every five nodes $\mathbf{p}, \mathbf{q}, \mathbf{r}, \mathbf{q}_{1}, \mathbf{r}_{1}$ of the graph $\Gamma$ such that

- the non-empty $S C C T_{S C C}\left(\mathbf{p}, \mathbf{q}, \mathbf{r}, \mathbf{r}_{1}\right)$ and $T_{S C C}\left(\mathbf{p}, \mathbf{r}, \mathbf{q}, \mathbf{q}_{1}\right)$ exist,

- the node $\left(\mathbf{p}, \mathbf{q}_{1}, \mathbf{r}_{1}\right)$ is an $S C C$-node of the graph $\Gamma^{3}$,

- $(\mathbf{q}, \mathbf{r}) \succeq\left(\mathbf{q}_{1}, \mathbf{r}_{1}\right)$ in $\Gamma^{2}$,

holds $T_{S C C}\left(\mathbf{p}, \mathbf{q}, \mathbf{r}, \mathbf{r}_{1}\right)=T_{S C C}\left(\mathbf{p}, \mathbf{r}, \mathbf{q}, \mathbf{q}_{1}\right)$.

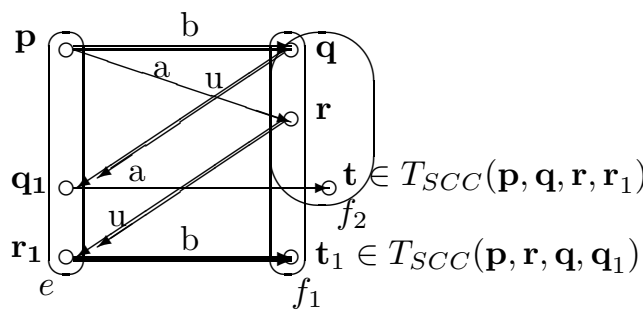




\section{Algorithm to verify the local threshold testability}

A linear depth-first search algorithm finding all $S C C$ of the given directed graph (see [1], [26] or [17]) will be used.

\subsection{To check the reachability on an oriented graph}

For a given node $\mathbf{q}_{\mathbf{0}}$, we consider depth-first search from the node. First only qo will be marked. Every edge is crossed two times. Given a node, the considered path includes first the ingoing edges and then the outgoing edges. After crossing an edge in the positive direction from the marked node $\mathbf{q}$ to the node $\mathbf{r}$ we mark $\mathbf{r}$ too. The process is linear in the number of edges (see [1], [15] for details).

The set of marked nodes forms a set of nodes that are reachable from $\mathbf{q}_{\mathbf{0}}$. The procedure may be repeated for any node of the graph $G$.

The time of the algorithm for all pairs of nodes is $O\left(n^{2}\right)$.

\subsection{To verify local threshold testability}

Let us find all $S C C$ of the graphs $\Gamma, \Gamma^{2}$ and $\Gamma^{3}$ and mark all $S C C$-nodes $\left(O\left(n^{3}\right)\right.$ time complexity).

Let us recognize the reachability on the graph $\Gamma$ and $\Gamma^{2}$ and form the table of reachability for all pairs of $\Gamma$ and $\Gamma^{2}$. The time required for this step is $O\left(n^{4}\right)$.

Let us check the conditions of lemma 13. For every $S C C$-node $(\mathbf{p}, \mathbf{q})(\mathbf{p} \neq \mathbf{q})$ from $\Gamma^{2}$ let us check the condition $\mathbf{p} \sim \mathbf{q}$. A negative answer for any considered node $(\mathbf{p}, \mathbf{q})$ implies the validity of the condition. In opposite case the automaton is not locally threshold testable. The time of the step is $O\left(n^{2}\right)$.

For every four nodes $\mathbf{p}, \mathbf{q}, \mathbf{r}, \mathbf{r}_{1}$ of the graph $\Gamma$, let us check the following conditions (see 16): $\mathbf{p} \succeq \mathbf{r} \succeq \mathbf{r}_{1}$ and $\mathbf{p} \succeq \mathbf{q}$. In a positive case, let us form $S C C T_{S C C}\left(\mathbf{p}, \mathbf{q}, \mathbf{r}, \mathbf{r}_{1}\right)$ of all nodes $\mathbf{t} \in \Gamma$ such that $\left(\mathbf{p}, \mathbf{r}_{1}\right) \succeq(\mathbf{q}, \mathbf{t})$ and $(\mathbf{q}, \mathbf{r}, \mathbf{t})$ is an $S C C$-node. In case that $S C C T_{S C C}$ is not well defined the automaton is not threshold testable. The time required for this step is $O\left(n^{5}\right)$.

For every five nodes $\mathbf{p}, \mathbf{q}, \mathbf{r}, \mathbf{q}_{1}, \mathbf{r}_{1}$ from $\Gamma$ let us check now the second condition of theorem 17 . If nonempty components $T_{S C C}\left(\mathbf{p}, \mathbf{q}, \mathbf{r}, \mathbf{r}_{1}\right)$ and $T_{S C C}\left(\mathbf{p}, \mathbf{r}, \mathbf{q}, \mathbf{q}_{1}\right)$ exist, the node $\left(\mathbf{p}, \mathbf{q}_{1}, \mathbf{r}_{1}\right)$ is an $S C C$-node of the graph $\Gamma^{3}$ and $(\mathbf{q}, \mathbf{r}) \succeq\left(\mathbf{q}_{1}, \mathbf{r}_{1}\right)$ in $\Gamma^{2}$, let us verify the equality $T_{S C C}\left(\mathbf{p}, \mathbf{q}, \mathbf{r}, \mathbf{r}_{1}\right)=T_{S C C}\left(\mathbf{p}, \mathbf{r}, \mathbf{q}, \mathbf{q}_{1}\right)$. If the answer is negative then the automaton is not threshold testable. A positive answer for all considered cases implies the validity of the condition of the theorem. The time required for this step is $O\left(n^{5}\right)$.

The whole time of the algorithm to check the local threshold testability is $O\left(n^{5}\right)$.

\section{The local testability}

We present now necessary and sufficient conditions of local testability of Kim, McNaughton and McCloskey ([15], [16]) in the following form:

Theorem 31 ([16]) A DFA with state transition graph $\Gamma$ and transition semigroup $S$ is locally testable iff the following two conditions hold:

1) For any $S C C$-node $(\mathbf{p}, \mathbf{q})$ from $\Gamma^{2}$ such that $\mathbf{p} \sim \mathbf{q}$ we have $\mathbf{p}=\mathbf{q}$.

2)For any $S C C$-node $(\mathbf{p}, \mathbf{q})$ from $\Gamma^{2}$ such that $\mathbf{p} \succ \mathbf{q}$ and arbitrary element $s$ from $S$ we have $\mathbf{p} s \succeq \mathbf{q}$ is valid iff $\mathbf{q} s \succeq \mathbf{q}$.

The theorem implies

Corollary 32 A DFA with state transition graph $\Gamma$ over alphabet $\Sigma$ is locally testable iff the following two conditions hold:

1)For any $S C C$-node $(\mathbf{p}, \mathbf{q})$ from $\Gamma^{2}$ such that $\mathbf{p} \sim \mathbf{q}$ we have $\mathbf{p}=\mathbf{q}$.

2)For any node $(\mathbf{r}, \mathbf{s})$ and any $S C C$-node $(\mathbf{p}, \mathbf{q})$ from $\Gamma^{2}$ such that $(\mathbf{p}, \mathbf{q}) \succ(\mathbf{r}, \mathbf{s}), \mathbf{s} \sim \mathbf{q}$ and for arbitrary $\sigma$ from $\Sigma$ we have $\mathbf{r} \sigma \succeq \mathbf{s}$ is valid iff $\mathbf{s} \sigma \succeq \mathbf{s}$. 


\section{Algorithm to verify the local testability}

In [16], a polynomial time algorithm for local testability problem was considered. Now we present another version of such algorithm with the same time complexity. We hope that it will be more simple.

Let us form a table of reachability on the graph $\Gamma\left(O\left(n^{2}\right)\right.$ time complexity).

Let us find $\Gamma^{2}$ and all $S C C$-nodes of $\Gamma^{2}$.

For every $S C C$-node $(\mathbf{p}, \mathbf{q})(\mathbf{p} \neq \mathbf{q})$ from $\Gamma^{2}$ let us check the condition $\mathbf{p} \sim \mathbf{q}$. $\left(O\left(n^{2}\right)\right.$ time complexity $)$. If the condition holds then the automaton is not locally testable (32).

Let us exclude all edges $(\mathbf{p}, \mathbf{q}) \rightarrow(\mathbf{r}, \mathbf{s})$ from the graph $\Gamma^{2}$ such that $\mathbf{s} \nsucceq \mathbf{q}$ and $\mathbf{s} \nsucceq \mathbf{p}$. Then let us mark all nodes $(\mathbf{p}, \mathbf{q})$ of the graph $\Gamma^{2}$ such that for some $\sigma$ from $\Sigma$ from the two conditions $\mathbf{p} \sigma \succeq \mathbf{q}$ and $\mathbf{q} \sigma \succeq \mathbf{q}$ only one is valid. The time required for this step is $O\left(n^{2}\right)$.

Then we add to the graph new node $(\mathbf{0}, \mathbf{0})$ with edges from this node to every $S C C$-node. Let us find the set of nodes reachable from the node $(\mathbf{0}, \mathbf{0}) .\left(O\left(n^{2}\right)\right.$ time complexity). The automaton is locally testable iff no marked node belongs to obtained set (32).

The whole time of the algorithm to check the local testability is $O\left(n^{2}\right)$.

\section{Acknowledgments}

I would like to express my gratitude to Stuart Margolis for posing the problem and for helpful suggestions on improving the style of the paper and to referees for important and useful remarks.

\section{References}

1. A. Aho, J. Hopcroft, J. Ulman, The Design and Analisys of Computer Algorithms, Addison-Wesley, 1974.

2. J. Almeida, Implicit operations on finite $J$-trivial semigroups and a conjecture of I. Simon, J. Pure Appl. Alg., 69(1990), 205-208.

3. M.-P. Beal, J. Senellart, On the bound of the synchronization delay of local automata, Theoret. Comput. Sci. 205, 1-2(1998), 297-306.

4. D. Beauquier, J.E. Pin, Factors of words, Lect. Notes in Comp. Sci, Springer, Berlin, 372(1989), 63-79.

5. D. Beauquier, J.E. Pin, Languages and scanners, Theoret. Comp. Sci, 1, 84(1991), 3-21.

6. J.-C. Birget, Strict local testability of the finite control of two-way automata and of regular picture description languages, J. of Alg. Comp., 1, 2(1991), 161-175.

7. J.A. Brzozowski, I. Simon, Characterizations of locally testable events, Discrete Math., 4, (1973), $243-271$.

8. P. Caron, LANGAGE: A Maple package for automaton characterization of regular languages, Springer, Lect. Notes in Comp. Sci., 1436(1998), 46-55.

9. Z. Esik, I. Simon, Modelling literal morphisms by shuffle. Semigroup Forum, 56(1998), 2, 225-227.

10. K.S. Fu, Pattern recognition and applications, Prentice Hall, N.Y., 1982.

11. A. Ginzburg, About some properties of definite, reverse-definite and related automata, IEEE Trans. Electron. Comput. EC-15(1966), 806-810.

12. R.C. Gonzales, M.G. Thomason, Syntactic pattern recognition. An introduction, Addison Wesley, N.Y., 1978.

13. T. Head, Formal languages theory and DNA: an analysis of the generative capacity of specific recombinant behaviors, Bull. Math. Biol., 49(1987), 4, 739-757.

14. F. Hinz, Classes of picture languages that cannot be distinguished in the chain code concept and deletion of redundant retreats, Springer, Lect. Notes in Comp. Sci., 349(1990), 132-143.

15. S. Kim, R. McNaughton, R. McCloskey, An upper bound for the order of locally testable deterministic finite automaton, Lect. Notes in Comp. Sci., 401(1989), 48-65.

16. S. Kim, R. McNaughton, R. McCloskey, A polynomial time algorithm for the local testability problem of deterministic finite automata, IEEE Trans. Comput. 40(1991) N10, 1087-1093.

17. S. Kim, R. McNaughton, Computing the order of a locally testable automaton, Lect. Notes in Comp. Sci, Springer, 560(1991), 186-211.

18. G. Lallement, Semigroups and combinatorial applications, Wiley, N.Y., 1979. 
19. S. W. Margolis, J.E. Pin, Languages and inverse semigroups, Lect. Notes in Comp. Sci, Springer, 199(1985), 285-299.

20. R. McNaughton, S. Papert, Counter-free automata, M.I.T. Press Mass., 1971.

21. M. Minsky, S. Papert, Perceptrons, M.I.T. Press Mass., 1971, Cambridge, MA, 1969.

22. J. Pin, Finite semigroups and recognizable languages. An introduction, Semigroups and formal languages, Math. and Ph. Sci., 466(1995), 1-32.

23. M. Perles, M. O. Rabin, E. Shamir, The theory of definite automata, IEEE Trans. Electron. Comput. EC12(1963), 233-243.

24. J. Reiterman, The Birkhoff theorem for finite algebras, Algebra Universalis, 14(1982), 1-10.

25. J. Ruiz, S. Espana, P. Garcia, Locally threshold testable languages in strict sense: Application to the inference problem. Springer, Lect. Notes in Comp. Sci., 1433(1998), 150-161.

26. R.E. Tarjan, Depth first search and linear graph algorithms, SIAM J. Comput., 1(1972), 146-160

27. W. Thomas, Classifying regular events in symbolic logic, J. of Comp. System Sci, 25(1982), 360-376.

28. A.N. Trahtman, The varieties of n-testable semigroups, Semigroup Forum, 27(1983), 309-318.

29. A.N. Trahtman, A polynomial time algorithm for local testability and its level. Int. J. of Algebra and Comp., v. 9, 1(1998), 31-39.

30. A.N. Trahtman, The identities of k-testable semigroups. Comm. in algebra, v. 27, 11(1999), 5405-5412.

31. A.N. Trahtman, A precise estimation of the order of local testability of a deterministic finite automaton, Springer, Lect. Notes in Comp. Sci., 1436(1998), 198-212.

32. E. Vidal, F. Casacuberta, P. Garcia, Grammatical inference and automatic speech recognition. In speech recognition and coding, Springer, 1995, 175-191.

33. Th. Wilke, Locally threshold testable languages of infinite words, Lect. Notes in Comp. Sci, Springer, Berlin, 665(1993), 63-79.

34. Th. Wilke, An algebraic theory for regular languages of finite and infinite words, Int. J. Alg. and Comput. 3(1993), 4, 447-489.

35. Y. Zalcstein, Locally testable semigroups, Semigroup Forum, 5(1973), 216-227.

36. Y. Zalcstein, Locally testable languages, J. Comp. System Sci., 6(1972), 151-167. 